\title{
Surface History of Mercury: Implications for Terrestrial Planets
}

\author{
Bruce C. Murray, ${ }^{1}$ Robert G. Strom, ${ }^{2}$ Newell J. Trask, ${ }^{3}$ and Donald E. Gault ${ }^{4}$
}

\begin{abstract}
A working hypothesis of Mercury's history is presented. We infer the surface of Mercury to record a sequence of events broadly similar to those recorded on the moon, implying similar histories of impact bombardment. The oldest terrains on Mercury seem to be better preserved from modification by ejecta from subsequently formed impact basins because of higher surface gravity. The large lunarlike impact craters on Mercury can be interpreted as part of a distinct episode of bombardment which may have affected all the terrestrial planets about 4 b.y. ago. The light cratering accumulated on the surfaces of the Mercurian smooth plains is similar in diameter/frequency relationship to that of the lunar maria and of the oldest Martian plains unjts, consistent with recent interpretations of lunar and Martian flux histories by Wetherill (1974) and Soderblom et al. (1974). A straightforward interpretation of the Mercurian surface record thus supports recent order of magnitude increases in age estimates of many Martian features discovered by Mariner 9 but is not conclusive. The large core inferred for Mercury combined with the lack of recognizable evidence of past atmospheric activity is more easily understood in terms of radially heterogeneous accumulation than in terms of differentiation of a homogeneous planet. Early core cooling may be reflected by widespread evidence of crustal shortening. However, Mercury's surface seems little affected by any tectonic, atmospheric, or volcanic processes for the last 3 b.y. or so, raising questions concerning (1) the relationship of the origin of Mercury's magnetic field to that of earth's and (2) the primary cause of volcanic flooding, which may have begun, and ended, approxımately synchronously on Mercury and the moon.
\end{abstract}

\section{INTRODUCTION}

Our goal in this paper is to portray a plausible history of Mercury as suggested by the Mariner 10 data and to call attention to the implications for the history of the other terrestrial planets. We view this first 'working hypothesis' for the history of Mercury not as a definitive statement but as an initial framework to stimulate and focus subsequent analyses and interpretation.

A convenient way to discuss the history of Mercury as suggested by the Mariner 10 television pictures is to postulate five periods delineated by successive variations in the modification of the surface by external and internal processes: (1) accretion and differentiation, (2) terminal heavy bombardment, (3) formation of the Caloris basin, (4) flooding of that basin and other areas, and (5) light cratering accumulated on the smooth plains.

The sequence of events recorded on Mercury's surface closely resembles that of the moon, although there are interesting differences in detail. Whether the absolute time periods represented by those sequences also are closely similar is one principal question in the interpretation of the Mariner 10 photography. The other major issue is whether the smooth plains of Mercury, like those of the moon, are primarily of volcanic origin. Our working hypothesis is that Mercury is indeed moonlike in both respects, absolute history and extensive volcanism, and we will outline the basis for our judgments. However, very divergent interpretations, including major differences in the early history of the two objects and even nonvolcanic origin of the plains, cannot be ruled out entirely on the basis of the analyses carried out so far.

\section{ACCretion AND Differentiation}

Strom et al. [1975a] have argued the case for at least some volcanic extrusions on Mercury of morphology similar to the

\footnotetext{
${ }^{1}$ Division of Geological Sciences, California Institute of Technology, Pasadena, California 91125.

${ }^{2}$ University of Arizona, Tucson, Arizona 85721

${ }^{3}$ U.S. Geological Survey, Reston, Virginia 22070.

- NASA/Ames Research Center. Mountain View, California 94040.

Copyrıght $(C) 1975$ by the American Geophysical Union.
}

lunar maria and of thousands of kilometers in horizontal dimension, emphasizing stratigraphic, geographic, volumetric, and albedo relationships. Figure 1 presents additional data supporting a volcanic rather than impact origin of the plains east of the Caloris basin. By analogy with the earth [Bowen, 1928] and the moon [Ringwood and Essene, 1970], large-scale volcanism on Mercury would imply the existence of a silicate mantle probably of at least several hundred kilometers in depth [Murray et al., 1974, note 20]. Yet the models of Reynolds and Summers [1969] indicate that a silicate outer shell of only 500 - or $600-\mathrm{km}$ thickness requires all the silicate available in a planet with a mean density of 5.45 . Hence on the basis of the television data alone it can be inferred that the planet is most likely composed of a large iron core enclosed by a relatively thin silicate layer from which the smooth plains originated.

The existence of an iron core on Mercury also has been inferred from the magnetic field observations [Ness et al., 1974, 1975]. Ness et al. argue that either an active dynamo or a fossil magnetic field must be involved. We would emphasize here that regardless of the origin of the magnetic field, a substantial iron core is indicated by Mercury's interaction with the solar wind. Thus the conclusion that Mercury is a differentiated planet is suggested by two independent observations.

Granted that Mercury very probably is composed of a large iron core and a silicate outer shell, when did that chemical segregation take place?

Regardless of how Mercury accumulated as a planet, chemical differentiation must have been complete well before any of the existing impact craters formed, since melting or any subsequent crustal plasticity would have modified the lunarlike appearance that they still retain. In addition, if any atmosphere was generated during or after accretion, it too must have disappeared entirely before any of the present topographic features formed. A very small amount of atmosphere will markedly modify the appearance of secondary craters and ejecta blankets surrounding a primary impact crater. For example, Mars, with an average surface pressure at present of only about 5 mbar, very rarely exhibits the superficial features characteristic of fresh impact craters on the moon and Mercury. Thus we conclude that profound chemical 


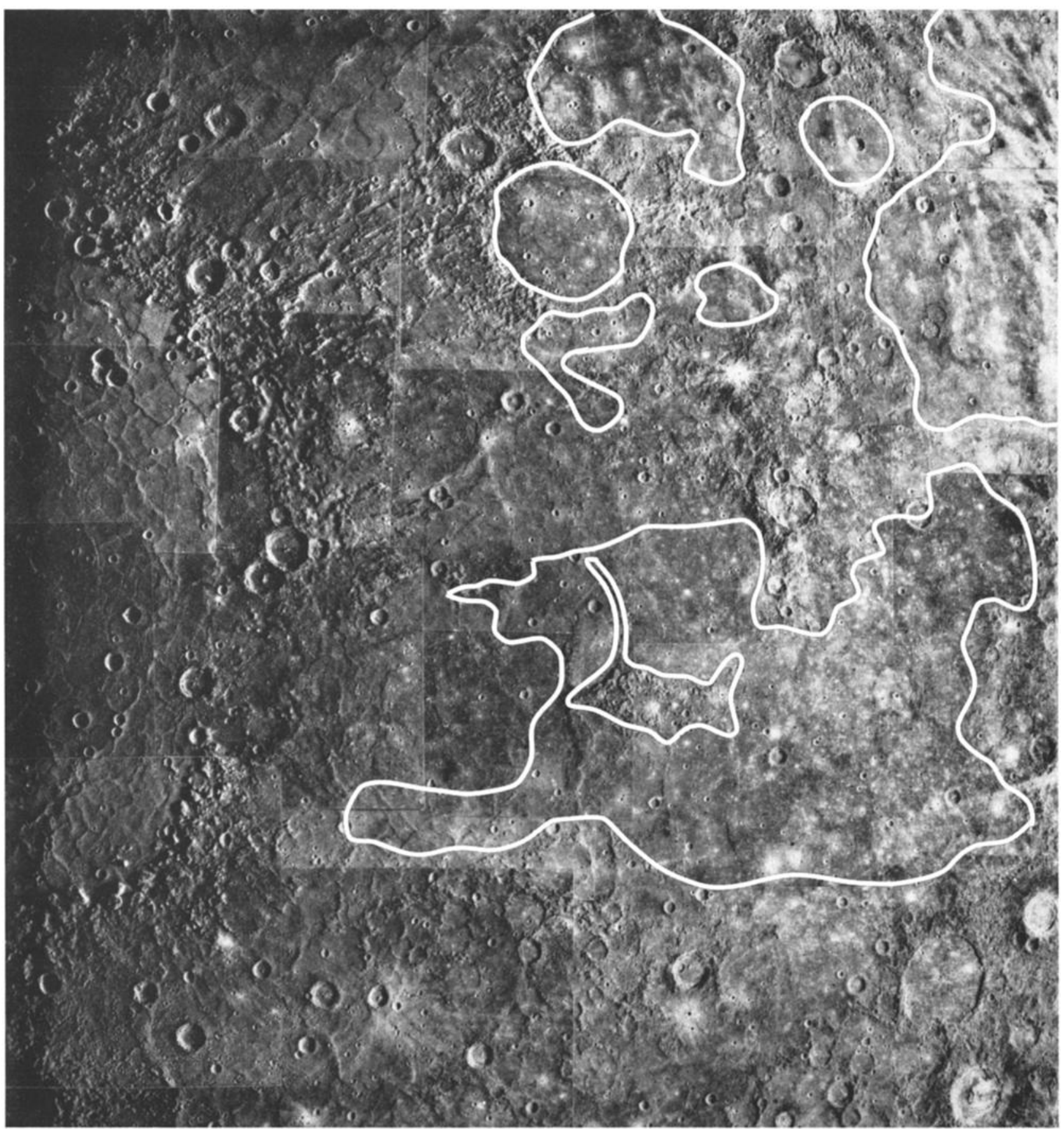

Fig. 1. Volcanic plains east of Caloris basin. In this photomosaic of the Caloris basin and adjacent areas, north is at the top. The area of Mercury shown is about $2000 \mathrm{~km}$ in dimension. On the basis of the distribution of large $(20-50 \mathrm{~km})$ fresh impact craters the smooth plains east of Caloris basin outlined in white are inferred by us to be younger than both the mountainous rim and the surrounding ejecta deposits, as well as the older plains material which fills Caloris basin. These relationships appear inconsistent with the possibility that the smooth plains in question could have originated as fluidized ejecta from the Caloris impact event. A later volcanic origin is indicated instead.

segregation into a large iron core and relatively thin silicate mantle took place sufficiently before the formation of the large craters, so that any lingering atmosphere had largely dissipated and a rigid lithosphere had come into existence.

\section{Terminal Heavy Bombardment}

A period of heavy bombardment is represented by the large craters hundreds of kilometers in diameter grading into large basins, including the Caloris basin itself. As is discussed by Trask and Guest [1975], large craters appear in many areas to be emplaced into an older host material, although in a few regions the surface appears more nearly saturated with large craters $(>50 \mathrm{~km})$. In most of the older terrains, macroscopically smooth intercrater areas are discerned which must in part predate the formation of those large craters (see Figure 2, upper left).

On the moon, by contrast, the architecture of the present surface is dominated by a heavy bombardment that ended about $4 \times 10^{9}$ years ago and by subsequent lava filling. Older surfaces saturated with large craters as well as small areas 
similar to the extensive intercrater plains of Mercury also can be recognized. Terminal bombardment relationships may be more evident on Mercury than on the moon because the greater surface gravity on Mercury restricts the areal coverage of the debris from the impact formation of the basin [Gault et al., 1975]. Also there may have been fewer large impacts in comparison to the greater surface area, although that point is not clear. In any case, the existence of large intercrater areas on Mercury implies that some earlier process obliterated nearly all topographic remnants of the saturation bombardment that necessarily constituted the final stages of planetary accumulation (accretion). Otherwise, a surface saturated by large crater forms would still survive (see Figure 2, lower left).

Just what crater obliteration process is recorded by that old smooth surface? Conceivably, it could be a relic from melting associated with accretion itself or a record of erosion from an
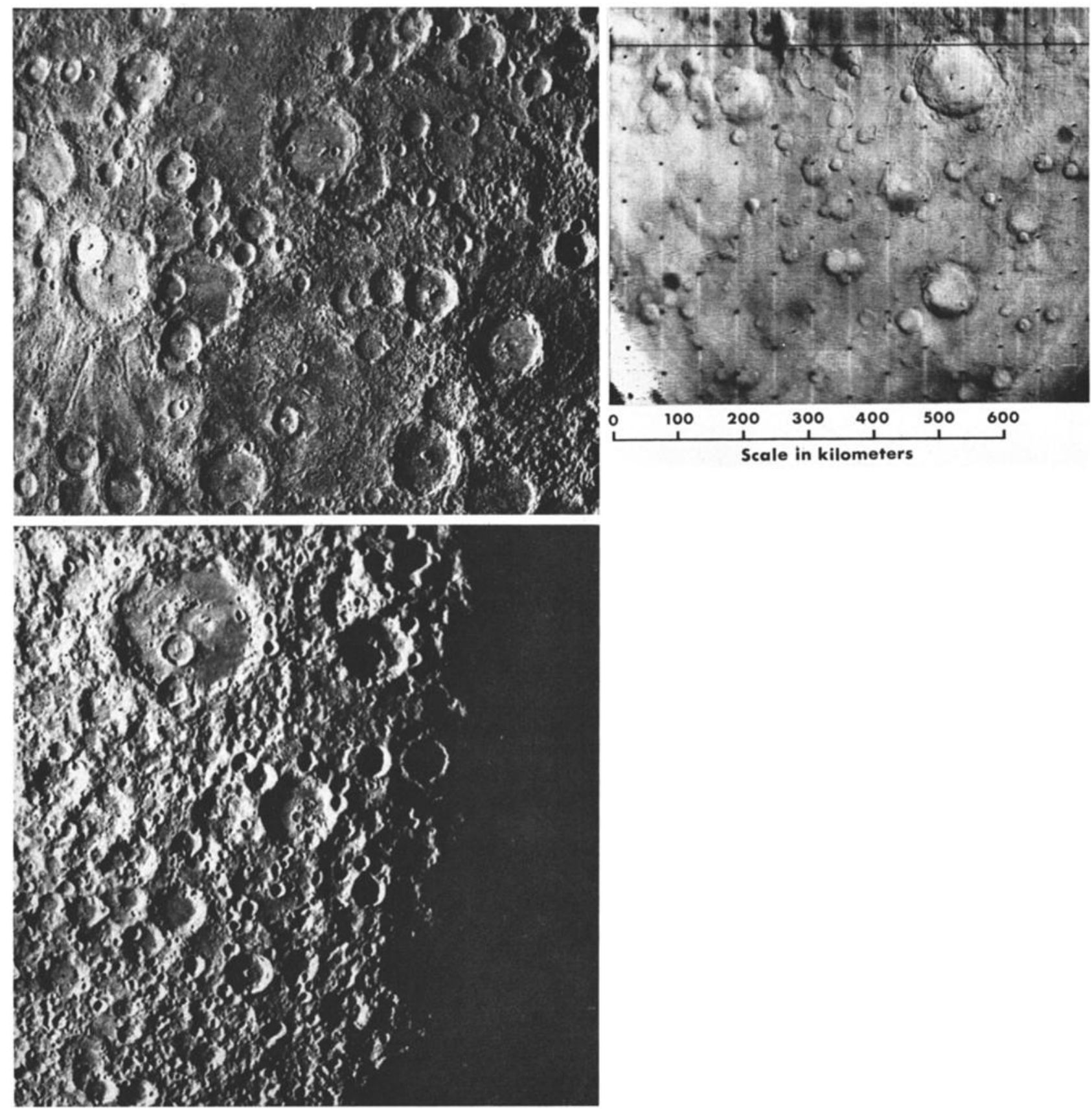

Fig. 2. Cratered terrains on Mercury, the moon, and Mars. (Upper left) Mariner 10 photograph of the bright-rayed crater Kuiper and surrounding cratered terrain on Mercury centered at $13{ }^{\circ} \mathrm{S}, 25^{\circ} \mathrm{W}$. The solar elevation angle varies from about $5^{\circ}$ to $27^{\circ}$ (Flight Data Subsystem 27,256). (Lower left) Mariner 10 photograph region in the northern uplands of the moon centered at $60^{\circ} \mathrm{N}, 130^{\circ} \mathrm{E}$. The solar elevation angle varies from $0^{\circ}$ (i.e., the terminator) to about $25^{\circ}$ (Flight Data Subsystem 2669). (Upper right) Mariner 9 photograph of cratered terrain in the Deuteronilus region on Mars centered at $30^{\circ} \mathrm{N}, 340^{\circ} \mathrm{W}$. The solar elevation angle varies from about $17^{\circ}$ to $33^{\circ}$ (DAS number $7,290,743$ ). All three images have been projected at the surface scale indicated. Both Mars and Mercury exhibit extensive intercrater plains, deficient in 10- to 50$\mathrm{km}$ craters in comparison with the moon. The Mercurian craters, however, resemble in distribution of morphology those of the moon rather than the highly degraded population present on Mars. From these relationships we infer a period of extensive crater obliteration on Mercury generally preceding the terminal heavy bombardment responsible for the present population of large craters on Mercury. 
associated atmospheric event. However, those processes should have operated globally, not leaving even slight remnants of saturated surfaces. For this reason, and on the basis of local morphology, we feel that it is somewhat more likely that the intercrater plains on Mercury constitute a very early volcanic surface. Petrologic data from the moon summarized by Albee [1975] suggest to some that there were early volcanic episodes on the moon now represented by occasional fragments in the breccias created by later bombardment. Another possible obliteration process is fluidized flow generated by large impacts, as was suggested recently for the origin of some smooth plains on the moon previously believed to be volçanic (D. E. Wilhelms, personal communication, 1975). Obliteration of early crater forms over some fraction of Mercury's surface by hypothetical catastrophic basin formation cannot entirely be ruled out at present. However, we consider the distribution and morphology of the Mercurian intercrater plains to be inconsistent with such an origin. In addition, the surface gravity of Mercury requires this cataclysmic process to be more localized on Mercury than on the moon.

It is useful at this point to consider Figure 2. The most heavily cratered areas of Mars also exhibit intercrater plains, i.e., also record a period of crater obliteration, as is shown in Figure 2, upper right. But in contrast to Mercury, most large Martian craters are characteristically modified, probably as a result of atmospheric erosion and deposition during and subsequent to the terminal heavy bombardment. The Mercurian craters (Figure 2, upper left), on the other hand, reflect the full distribution of morphologies seen on the moon (Figure 2, lower left); i.e., they record merely the normal degradation associated with the impact process itself.

This crucial question of the distribution of morphologies of the Mercurian and lunar craters warrants, and surely will receive, a major quantitative effort (which is now made much more tractable by recent success in creating useful stereo pairs from originally isolated Mariner 10 frames). In the meantime, careful examination of many individual Mariner 10 photographs has failed to reveal to us any consistent difference in the distribution of lunar and Mercurian crater morphologies other than the difference identified by Gault et al. [1975] as arising from the difference in surface gravity. As a consequence, we infer that the craters formed during the final stages of accretion had been nearly completely erased before the present population of large lunarlike craters was formed. Otherwise, there would still be present an obvious, even dominant, population of degraded crater forms, as there is on Mars, reflecting the persistence of the crater obliteration process. This means that if the bombardment presently recorded on Mercury were simply the end of a continuous bombardment process persisting since accretion itself, the process of crater obliteration must have (1) operated at a rate comparable to that of crater formation and (2) terminated abruptly. It seems difficult to require hypothetical melting, early volcanism, or atmospheric erosion to cease so abruptly. Conversely, the impacting flux recorded on Mercury may not have been continuous since accretion; instead there may have been an extensive period of crater obliteration by volcanic or other processes followed by a distinct episode of late bombardment. Most important, an episodic early history of Mercury is required either way. We find an episodic bombardment history more plausible at this point than the specially constrained episodic obliteration history which would be required if the bombardment had been continuously decreasing from accretion. Accordingly, we interpret the intercrater plains as recording one or more periods of crater obliteration separating the main accumulation of the planet from a heavy terminal bombardment. Obviously, future studies can be expected to challenge this interpretation and to search for an alternate explanation based, perhaps, on an episodic crater removal process such as catastrophic impact ejecta.

Another aspect of the heavily cratered terrains on Mercury suggests significant differences from the moon (and also from Mars). The intercrater areas especially exhibit compressional features, probably thrust or high-angle reverse faults [Strom et al., 1975a]; crustal shortening must have been taking place during the terminal heavy bombardment but evidently not much after that. Strom et al. suggest a net crustal shortening during this period corresponding to a few tenths of a percent reduction in the radius of Mercury. In contrast, significant crustal shortening on a global scale has not been recognized on the moon or Mars. Since the core mantle boundary is apparently quite shallow on Mercury and the proportional core volume is so large in comparison with the core volumes of the moon and Mars, it is plausible that these unique compressional features correspond to an early period of minor core shrinkage. Strom et al. note that a phase change from liquid to solid iron involving less than $6 \%$ of a large core, or even just the consequence of a higher coefficient of thermal expansion of solid metal compared to solid silicate, may be sufficient to produce the necessary radius decrease.

Thus the oldest terrains on Mercury probably represent remnants of an early period of crater obliteration followed by a distinct episode of terminal heavy bombardment. Crustal shortening was taking place at that time, perhaps corresponding to core shrinkage.

\section{Formation of Caloris Basin}

The impact which created the Caloris basin was a major event in the history of Mercury. It modified the landscape over much of the illuminated hemisphere observed by Mariner 10 and certainly affected the other hemisphere as well. In association with basin excavation a ring of mountains resembling the lunar Apennine mountains and hummocky terrain similar to that seen in the Orientale basin on the moon formed. Orientale is a particularly useful lunar ringed basin with which to compare because it was not flooded by extensive lava filling afterward, like most other lunar basins. Strom et al. show that many characteristic features of a lunar ringed basin also can be recognized around Caloris. Accordingly, all these features are ascribed to a single time interval associated with the Caloris basin impact.

It is also possible to correlate speculatively the peculiar hilly and lineated terrain recognized on the incoming hemisphere of the Mercury I photography with the Caloris basin event. That terrain is apparently restricted to a location approximately antipodal to Caloris: none was observed in the south polar region in the Mercury 2 photography [Strom et al., 1975b] nor on the outgoing hemisphere as viewed in the first encounter. Furthermore, local sequence relationships indicate that the terrain must have developed near the end of heavy bombardment [Strom et al., 1975a], i.e., approximately at the time of the Caloris impact. Schultz and Gault [1975] have speculated that the lineated and hilly terrain is the result of the focusing of the seismic energy from the Caloris impact at the antipodal point and therefore the terrain formed at the time of the Caloris impact. Smaller areas of similar terrain have been recognized antipodal to the Imbrium and to the Orientale basins on the moon and attributed to focusing of ejecta rather than seismic 
energy [Moore et al., 1974]; this mechanism is inhibited in the case of Mercury by the higher surface gravity. The less obvious nature of the lunar occurrences is consistent with the relationship discussed by Gault et al. [1975] that the material between ancient basins is more visible on Mercury because the basin ejecta was restricted to narrower rings.

\section{Basin Flooding}

The smooth plains of much of the outgoing hemisphere and parts of the incoming hemisphere of Mercury represent a secondary filling of earlier basins, generally at a time when the impact flux had greatly decreased. There is plausible evidence [Trask and Guest, 1975; Strom et al., 1975a] that filling was not simultaneous and that much of the fill must be of volcanic origin, rather than from ejecta sheets, impact melt, or mass wasting processes (also see Figure 1). Nevertheless, the total time period represented by these plains compared to the total time interval recorded on the surface of Mercury may be relatively brief. No difference as large as $1: 1.5$ in crater density of the various Mercurian plains in the equatorial regions has been recognized so far [Murray et al., 1974].

In addition to these planet-wide filling episodes, probably relatively close in time but nevertheless discrete, there was a subsequent structural modification of the Caloris basin itself. Deformation continued after the end of filling, resulting in open fractures now several kilometers across which cut across earlier ridges to comprise the present surface. This pattern of tensional deformation is localized to Caloris basin: whether it was a subsequent uplift or subsidence, it can be ascribed plausibly to adjustment to the principal Caloris event [Strom et al., 1975a].

\section{Post-Filling Period}

The last and presumably longest episode recorded on the surface of Mercury is represented by the light cratering present over the entire planet and especially apparent on the smooth plains. Bright-rayed craters are very common and tend to be bluish in color in comparison to the underlying material [Hapke et al., 1975]. They resemble in morphology and in size-frequency spectrum the post-mare cratering of the moon; the Mercurian crater-frequency relationships measured so far all plot between those of the Apollo 12 and Apollo 14 sites [Murray et al., 1974]. Thus since the formation of the plains, Mercury has accumulated a light cratering similar to that of the moon.

Equally significant is the absence of substantial surface modification arising from tectonic, volcanic, or atmospheric processes. No tangible atmosphere accumulated sufficiently to transport fine particles, nor has there been any observable deformation of the silicate outer shell of Mercury arising from motions of any hypothetical fluid core. The heat machine apparently was turned off after formation of the plains, and that was the end of it, although future exploration of the $50 \%$ of the Mercurian surface not visible to Mariner 10 could reveal more recent episodes of localized internal activity.

Thus to us the most plausible sequence of events recorded on the surface of Mercury is (1) major chemical differentiation either during or very shortly after accretion, (2) after decline of the original bombardment, a period of crater obliteration, perhaps through extensive volcanism, followed by an episode of terminal heavy bombardment, global crustal shortening taking place at this time, (3) formation of the Caloris basin near the end of terminal bombardment, (4) volcanic flooding of large areas of the planet to form most of the smooth plains, and (5) light cratering subsequent to the filling episodes re- cording an accumulated flux comparable to that recorded on the lunar maria.

Other interpretations of Mercury's history are not ruled out by the Mariner 10 data, although we consider them less plausible. For example, the crater obliteration process might have been catastrophic, such as might accompany early large basin formation. In that case the terminal heavy bombardment need not have been episodic. It is also still possible, in the absence of truly definitive morphological evidence, that none of the smooth plains are of volcanic origin, although we think it quite unlikely.

\section{Comparison With Surface Histories of Venus, THE MoON, AND MARS}

Studies of returned lunar samples have demonstrated that heavy bombardment occurred on the moon as late as $\mathbf{5 0 0}$ m.y. after accretion [Tera et al., 1974]. However, there is not a consensus as to whether such bombardment was episodic [Stewart, 1975] or continuous and whether it was an event restricted to the moon or was solar system wide [Hartmann, 1975; Wetherill, 1975]. The Mercury results seem relevant to both questions. The terminal heavy bombardment recorded on Mercury probably could not have occurred appreciably more recently than the emplacement of the oldest lunar mare surfaces ( $\sim 3.8$ b.y. ago); it is difficult to imagine a population of large objects (i.e., capable of producing the Caloris basin) impacting Mercury long after accretion which would not also have impacted the moon. Most important, episodic terminal bombardment evidently occurred on Mercury. Thus we find it plausible to correlate the terminal bombardments on both the moon and Mercury as resulting from a distinct episode that affected at least the inner solar system about 4 b.y. ago.

There still remains the seemingly contrived possibility that the Mercurian surface records an earlier episodic bombardment and the moon records a later heavy bombardment which somehow did not affect Mercury. But Mars also exhibits a heavily cratered surface (in equatorial and southern hemispheric areas) which strikingly resembles the Mercurian cratered terrain; i.e., it is only partially saturated with large craters (see Figure 2). Very large ringed basins also have been recognized [Wilhelms, 1973]. The simplest and, to us, the most plausible explanation is that all three surfaces record the same episode of solar system bombardment, presumably from objects originating in large aphelia orbits. It is not possible, however, on the basis of the picture data alone (i.e., ignoring dynamical considerations), to exclude entirely the possibility that the old cratered surfaces instead record late bombardment of accretionary objects, objects in heliocentric orbits similar to those of the three planets.

If a terminal heavy bombardment affected both Mercury and the moon, then Venus must have been bombarded as well. Recent radar images of portions of the equatorial regions of Venus [Rumsey et al., 1974] show large circular craterlike topographic forms in some areas. If these features prove upon higher-resolution examination to be of probable impact origin, then they very probably have survived at least $4 \times 10^{\circ}$ years, since any more recent episode of bombardment should have affected Mercury and the moon as well. Such a conclusion is most significant, since it would mean that Venus, despite its similarity to earth in size and mass, has not manifested a global tectonic and volcanic style sufficient to obliterate all such ancient topography. Similarly, severe constraints on the erosional capacity of the extremely dense hot Venus atmosphere would be implied. 
Extensive areas on the moon and apparently on Mercury were flooded by volcanism after the end of late heavy bombardment, and internal activity on both planets evidently ceased after that volcanic episode. (Similar volcanic flooding also occurred on Mars following the end of heavy bombardment [Soderblom et al., 1974; Wilhelms, 1973] but continued longer, along with subsequent shield volcanism and extensional deformation.) The timing and mechanism of volcanic flooding on the moon has been related to specific models of lunar thermal history and partial melting. (For a review of these ideas, see Albee [1975].) In view of the profoundly different structure of the Mercurian interior as compared to that of the moon, it seems most fortuitous for a closely similar history of surface igneous activity, and cessation of activity, to have developed on both Mercury and the moon. It can be speculated that somehow the subsequent volcanic flooding on both planets (and perhaps Mars as well) is a delayed but direct consequence of the heavy bombardment itself. We can offer no plausible mechanism, however, to explain such a delayed effect. Alternatively, in the unlikely (to us) event that the smooth plains of Mercury are determined confidently to be entirely of impact, rather than volcanic, origin, then the difficulties raised by the apparent similarities in igneous history of the moon and Mercury would be avoided.

It is quite significant that the light cratering on the flooded plains of Mercury is similar to that on the maria of the moon in diameter-frequency relationships; similar flux histories over the last 3-4 b.y. are implied if the Mercurian surfaces are of comparable age. Relatively uniform impact flux histories throughout the inner solar system for the last 3-4 b.y. were inferred recently by Wetherill [1974], who concluded that the impacting objects probably originated in large aphelia orbits (like comets) rather than directly from the asteroid belt. Similar flux histories for Mars and the moon were independently hypothesized by Soderblom et al. [1974] on the basis of the similarity of diameter-frequency versus areal coverage relationships for those two objects. In contrast, many earlier analyses had hypothesized that the apparent impact flux on Mars was 6-20 times greater than that which had affected the moon [Hartmann, 1973, 1966; Anders and Arnold, 1965]. As a result, the age of the large shield volcano Olympus Mons, for instance, was initially placed at $40 \mathrm{~m} . y$. with an uncertainty of a factor of 2 or 3 [McCauley et al., 1972]. The same diameter-frequency data now would be inferred to indicate by the Soderblom-Wetherill hypothesis an average age of the surface materials closer to $500 \mathrm{~m}$.y. Similarly, the oldest cratered plains on Mars, which strongly resemble the lunar maria in morphology, albedo, and degree of cratering, would now be deemed to be of comparable age as well, i.e., 3-4 b.y.

It is still possible, of course, that the Mercurian plains are older than the lunar maria, just enough older to have accumulated virtually all of their present crater populations sufficiently close to the peak of the terminal heavy bombardment episode to mimic the flux accumulated on the lunar maria over $3 \frac{1}{2}$ b.y. Only under such coincidental conditions can the lunar and Martian plains crater populations be ascribed to a source of objects exhibiting strong depletion between the orbits of Mars and Mercury, and the Martian surfaces consequently be assigned ages much younger than comparably cratered lunar surfaces.

\section{IMPLICATIONS FOR EARTH}

The Mercury pictures support the notion that a late heavy bombardment, dated from lunar samples at about 4 b.y., was an episode which characterized the entire inner solar system. The earth therefore very probably underwent a comparable bombardment as well. Such a conjecture is not new [Safronov, 1972]. However, now the bombardment event 4 aeons ago must be regarded as being most probable and included in any reconstruction of the earth's history.

What would be the effects of major basin-forming impacts on earth? Imbrium and Caloris were probably excavated initially to a depth of $100-200 \mathrm{~km}$; permanent modification of the host material certainly transpired to some appreciably greater depth. Such events on earth 4 b.y. ago must have created physical and chemical heterogeneities in the upper mantle. The oldest confidently dated rocks are 3.7-3.9 b.y. old [Goldich and Hedge, 1974; Moorbath et al., 1973]. Whether the absence of earlier samples reflects a planet-wide metamorphism, as it does on the moon, or is merely a matter of chance survival is an open question. In any case, the Mercury picture data, combined with lunar sample analyses, serve to emphasize that planetary history and geological history are almost convergent nearly 4 b.y. ago. Continuing study of ancient terrestrial rocks, as well as eventual sample return from other terrestrial planets, may help unite the two subjects firmly.

The existence of a large iron core, perhaps one even sustaining a currently active dynamo process [Ness et al., 1975], also carries implications for the origin and history of earth's core. The formation of earth's core is sometimes linked to a concurrent exhalation of volatiles to produce much of earth's primary atmosphere during planet-wide differentiation following homogeneous accretion [Ringwood, 1966]. The present terrestrial atmosphere is then ascribed to a long-term degassing. Such a process is not excluded for Mercury by the Mariner 10 data but is burdened by the need both to develop a thick cool lithosphere and to remove entirely any secondary atmosphere on Mercury before the end of heavy bombardment.

Atmospheric escape is governed by solar irradiation and by surface gravity; Mercury's nearness to the sun, in comparison to Mars, enhances certain atmospheric escape processes, for example. However, Mercury and Mars have similar surface gravities, so that differential solar irradiation would have to be afforded extraordinary effectiveness in governing the very different atmospheric histories of the two planets if Mercury ever evolved even minor amounts of volatiles to its surface. Conversely, if Mercury accumulated heterogeneously, i.e., with very early differentiation, then volatiles may never have accumulated on the surface of Mercury in significant amounts. (See, for example, Anderson [1973] and Kaula [1975].) Generally, we find heterogeneous accumulation to be more probable for Mercury and, by analogy, for the earth and the other terrestrial planets.

Regardless of how Mercury formed, very probably it has been a differentiated planet for well over 4 b.y., the inferred age of the large impact craters still preserved on its surface. Yet there is no evidence after the end of heavy bombardment of any modification of the silicate outer layers by 'hot spots' or fluid convection within that core. In contrast, hot spots and convection in the terrestrial mantle have been attributed speculatively to an origin associated with the core-mantle boundary. Of course, Mercury's core may have cooled to a stable solid configuration by the end of heavy bombardment; indeed the lobate scarps may record the end of that process. If so, Mercury's present magnetic field could not easily be attributed to an active dynamo field, the explanation preferred by Ness et al. [1975]. Likewise, there may be some difficulties in finding a plausible thermal and electrical model of Mercury to 
permit a 'fossil' magnetic field to survive 4 b.y. Such difficulties in finding any plausible interpretation of the Mercury magnetic field perhaps may be interpreted as providing new clues to otherwise ad hoc circumstances hypothecated within the earth's core to account for earth's magnetic field.

Mariner 10's voyage of exploration to the unknown planet Mercury has enlarged our knowledge not just of that planet but of the family of terrestrial planets, including our own.

Acknowledgments. An earlier version of this manuscript was thoroughly and constructively reviewed by Clark Chapman and William Hartmann of the Planetary Science Institute, Tucson, Arizona by William Kaula of the University of California at Los Angeles; and by Don Wilhelms of the U.S. Geological Survey, Menlo Park, California. As a consequence the paper has been substantially rewritten to present our interpretations more clearly and to call attention more conspicuously to alternative possibilities. We feel that the resulting paper has been substantially improved through response to the reviewers' efforts and wish to express our appreciation for their efforts. Contribution 2579 of the Division of Geological and Planetary Sciences, California Institute of Technology, Pasadena, California 91125 .

\section{REFERENCES}

Albee, A. L., A review of lunar sample studies and their application to studies of the terrestrial planets, Rev. Geophys. Space Phys., in press, 1975 .

Anders, E., and J. Arnold, Age of craters on Mars, Science, 149, 1494, 1965.

Anderson, D. L., The moon as a high temperature condensate, Moon, 8, 33-57, 1973.

Bowen, N. L., The Evolution of the Igneous Rocks, Princeton University Press, Princeton, N. J., 1928.

Gault, D. E., J. E. Guest, J. B. Murray, D. Dzurisin, and M. C. Malin, Some comparisons of impact craters on Mercury and the moon, $J$. Geophys. Res., 80, this issue, 1975.

Goldich, S. S., and C. E. Hedge, 3800-Myr granite gneiss in southwestern Minnesota, Nature, 252, 467, 1974.

Hapke, B., G. E. Danielson, Jr., K. Klaasen, and L. Wilson, Photometric observations of Mercury from Mariner 10, J. Geophys. Res., 80, this issue, 1975.

Hartmann, W. K., Martian cratering, Icarus, 5, 565, 1966.

Hartmann, W. K., Martian cratering, 4, Mariner 9 initial analysis of cratering chronology, J. Geophys. Res., 78, 4096, 1973.

Hartmann, W. K., Lunar cataclysm: A misconception?, Icarus, in press, 1975

Kaula, W. H., The seven ages of a planet, submitted to Icarus, 1975.

McCauley, J. F., M. H. Carr, J. A. Cutts, W. K. Hartmann, H. Masursky, D. J. Milton, R. P. Sharp, and D. E. Wilhelms, Preliminary Mariner 9 report on the geology of Mars, Icarus, 17 289, 1972.

Moorbath, S., R. K. O'Nions, and R. J. Parkhurst, Early Archaean age for the Isua iron formation, West Greenland, Nature, 245, 138 , 1973.

Moore, H. J., C. A. Hodges, and D. H. Scott, Multi-ring basins (il- lustrated by Orientale and associated features), Proceedings of the 5th Lunar Science Conference, Geochim. Cosmochim. Acta, Suppl. 5, 1, 71-100, 1974

Murray, B. C., M. J. S. Belton, G. E. Danielson, M. E. Davies, D. E Gault, B. Hapke, B. O'Leary, R. G. Strom, V. Suomi, and N. Trask, Mercury's surface: Preliminary description and interpretation from Mariner 10 pictures, Science, 185, 169, 1974.

Ness, N. F., K. W. Behannon, R. P. Lepping, Y. C. Whang, and K. H Schatten, Magnetic field observations near Mercury: Preliminary results from Mariner 10, Science, 185, 151, 1974.

Ness, N. F., K. W. Behannon, R. P. Lepping, and Y. C. Whang, Magnetic field of Mercury confirmed, submitted to Nature. 1975.

Reynolds, R. T., and A. L. Summers, Calculations on the composition of the terrestrial planets, J. Geophys. Res., 74, 2494, 1969.

Ringwood, A. E., Chemical evolution of the terrestrial planets, Geochim. Cosmochim. Acta, 30, 41, 1966.

Ringwood, A. E., and E. Essene, Petrogenesis of Apollo 11 basalts: Internal constitution and origin of the moon, Proceedings Apollo 11 Lunar Science Conference, Geochim. Cosmochim. Acta. Suppl. 1,1 , 769, 1970.

Rumsey, H. C., G. A. Morris, R. R. Green, and R. M. Goldstein, A radar brightness and altitude image of a portion of Venus, Icarus, $23,1,1974$.

Safronov, V. S. The initial state of the earth and certain features of its evolution, Izv. Acad. Sci. USSR Phys. Solid Earth, no. 7, 444, 1972.

Schultz, P. H., and D. E. Gault, Seismic effects from major basin formation on the moon and Mercury, NASA Tech. Memo. TM X-62, 388, 1974. (Also Moon, in press, 1975.)

Soderblom, L. A. C. D. Condit, R. A. West, B. M. Herman, and T. J. Kreidler, Martian planetwide crater distribution: Implications for geologic history and surface processes, Icarus, 22, 239, 1974.

Stewart, D. B., Apollonian metamorphic rocks-The products of prolonged subsolidus equilibration, in Lunar Science VI, Lunar Science Institute, Houston, Tex., 1975.

Strom, R. G., N. J. Trask, and J. E. Guest, Tectonism and volcanism on Mercury, J. Geophys. Res., 80, this issue, 1975a.

Strom, R. G., B. C. Murray, M. J. S. Belton, G. E. Danielson, M. E. Davies, D. E. Gault, B. Hapke, B. O'Leary, N. Trask, J. E. Guest, J. Anderson, and K. Klaasen, Preliminary imaging results from the second Mercury encounter, J. Geophys. Res., 80, this issue, 19756 .

Tera, F., D. A. Papanastassiou, and G. J. Wasserburg, Isotopic evidence for a terminal lunar cataclysm, Earth Planet. Sci. Lett., 22, $1,1974$.

Trask, N. J., and J. E. Guest, Preliminary geologic/terrain map of Mercury, J. Geophys. Res., 80, this issue, 1975.

Wetherill, G. W., Problems associated with estimation of the relative impact rate on Mars and moon, Moon, 9, 227, 1974.

Wetherill, G. W., Pre-mare cratering and early solar system history, in Proceedings of the Soviet-American Conference on Cosmochemistry of Moon and Planets, NASA, Greenbelt, Md., 1975.

Wilhelms, D. E., Comparison of Martian and lunar multiringed circular basins, J. Geophys. Res., 78, 4084, 1973.

(Received February 14, 1975;

revised March 3, 1975;

accepted March 5, 1975.) 